\title{
SUSTENTABILIDAD DE LOS SISTEMAS DE PRODUCCIÓN AGROPECUARIA
}

\author{
SUSTAINABILITY OF AGRICULTURAL PRODUCTION SYSTEMS \\ Rene Antonio Hinojosa Benavides ${ }^{*}$, (D) Rebeca Vitor Flores ${ }^{1}$, (D) Julio César Gonzales López ${ }^{1}$, \\ (D) Yony Quispe Rimachi' ${ }^{1}$ (D) Romel Angel Molina Huailla ${ }^{1}$, (D) Jerry Toñito Ricra Naupari', \\ (iD) Edgar Saul Sánchez Montes ${ }^{1}$, (D) Jhon Quispe de la Cruz ${ }^{1}$ \\ rhinojosa@unah.edu.pe; rebecavirgo1999@gmail.com; jucer18111994@gmail.com; 1712810219@unah.edu.pe; \\ 1712810214@unah.edu.pe; 1812810251@unah.edu.pe;1812810255@unah.edu.pe; 1812810249@unah.edu.pe \\ 1Universidad Nacional Autónoma de Huanta, Ayacucho, Perú \\ *Correspondencia: Rene Antonio Hinojosa Benavides. Email: rhinojosa@unah.edu.pe
}

Recibido: 06.11.19 | Aprobado: 29.11.19

\section{RESUMEN}

El objetivo fue analizar conceptos vinculados a la sustentabilidad de los sistemas de producción agropecuaria, reflejados en sus dimensiones ecológica, social y económica. La sustentabilidad refleja prosperidad económica sostenible en el tiempo, con calidad de vida, protegiendo paralelamente los sistemas naturales del planeta. Se concluyó que el desarrollo sustentable enmarca la capacidad que haya desarrollado el humano para satisfacer las necesidades de las generaciones presentes sin comprometer las oportunidades para el desarrollo de generaciones futuras y que la actividad agropecuaria constituye un problema ecológico, por lo que se requiere evaluar la eficiencia de sus sistemas en relación con su sustentabilidad.

Palabras clave: Sustentabilidad, sistemas de producción, agropecuaria.

\section{ABSTRACT}

The aim was to analyze concepts related to the sustainability of agricultural production systems, reflected in their ecological, social and economic dimensions. Sustainability reflects sustainable economic prosperity over time with quality of life, while protecting the natural systems of the planet. It was concluded that sustainable development frames the capacity that human has developed to meet the needs of present generations without compromising opportunities for the development of future generations and agricultural activity is an ecological problem, so it is necessary to evaluate the efficiency of their systems in relation to their sustainability.

Keywords: Sustainability, production systems, agricultural. 


\section{INTRODUCCIÓN}

Se define a la sustentabilidad como la habilidad de un sistema de mantener la productividad aun cuando sea sometido a "stress o perturbaciones" (Conway, 1994; citado por Bocero, 2002). A esta definición Chiappe y Piñeiro (2000; citados por Bolívar, 2011) agregaron que la agricultura sustentable implica el manejo y conservación de los recursos naturales conjuntamente con la orientación de cambios tecnológicos para asegurar la satisfacción continua de la generación humana presente y futura, puesto que tal desarrollo sustentable conserva el suelo, el agua y los recursos genéticos animales y vegetales; no degrada al medio ambiente; es técnicamente apropiado, económicamente viable y socialmente aceptable; y de esta definición Bautista y Smit (2012) destacan elementos referentes a la temporalidad, contexto global, generaciones futuras de los seres vivos, al agregarle la dimensión cultural integrada por las experiencias y vivencias documentadas en las memorias comunitarias campesinas indígenas cuya cosmovisión se inspira en el respeto a los recursos naturales.

El Análisis de los sistemas de producción agropecuaria en el marco de la sustentabilidad se centra primordialmente a nivel de unidad de producción agropecuaria ya que en este ámbito es donde se manifiesta claramente las debilidades y/o fortalezas de dicha producción, lo que permite una mejor discusión sobre sus barreras tanto técnicas como sociales, y una mejor comprensión de los efectos de la intervención del hombre sobre el ecosistema (Merma y Julca, 2012; Altieri et al. 2011). La intervención del hombre sobre la naturaleza constituye un desafío para todos los actores del sistema agropecuario. Hoy se realizan esfuerzos para unir sistemas regionales y nacionales de ciencia y tecnología, con el fin de promover una mejor calidad social y ambiental en los procesos de urbanización. Estos intentos han implicado la formalización y aceleración del aprendizaje individual, colectivo e institucional, mediante redes y espacios de colaboración, en aras de definir acciones dirigidas hacia un mejor desarrollo poblacional y urbano, bajo los principios de la sustentabilidad (Torres y Cruz, 2019).

\section{INTENSIFICACIÓN DE LOS SISTEMAS AGROPECUARIOS}

La creciente demanda alimentaria, como consecuencia de la creciente población mundial con su acelerado desarrollo económico, presiona constantemente los actuales sistemas de producción agropecuaria e impulsa cambios tecnológicos para satisfacer las nuevas exigencias productivas. Las actividades agropecuarias producen bienes y servicios indispensables para el ser humano y son una importante fuente de mano de obra (FAO, 2014); sin embargo, estas actividades pueden generar efectos negativos sobre el ambiente y la sociedad, reduciendo la sustentabilidad del uso del suelo (Vega et al., 2015). La horticultura es una actividad intensiva que hace un alto uso de pesticidas (Andrade, 2017). La necesidad de obtener ingresos a corto plazo y el uso de tecnologías inapropiadas por los agricultores, viene ocasionando la pérdida de recursos especialmente suelo y vegetación.

Es necesario cuantificar los daños producidos en los ecosistemas (Merma y Julca, 2012), ya que la aplicación de prácticas convencionales de manejo de cultivos que degradan los recursos naturales, han ocasionado múltiples consecuencias, entre ellas: contaminación de recursos naturales (suelo y agua), efectos nocivos e irreversibles sobre 
la salud del trabajador rural y su familia, del consumidor, de la población y del ambiente en general (Altieri, 2002 citado por Andrade, 2017). Los análisis convencionales que se utilizan para determinar los resultados de los sistemas agropecuarios (ej. costo/beneficio) no son adecuados para evaluar su funcionamiento a largo plazo, ya que no integran al análisis las dimensiones sociales y ambientales, las cuales adquieren cada día mayor relevancia (Flores y Sarandón, 2004) citados por Albicette et al., 2009)

La producción de alimentos en el campo y el desarrollo sostenible deben coexistir para reducir el deterioro de nuestro planeta. Los sistemas de producción agrícolas, pecuarios o agropecuarios están basados en características específicas, que se derivan de la diversidad existente de los recursos y a las particularidades de los productores (Martínez, 2009). Los sistemas de granjas individuales que en conjunto presentan una base de recursos, patrones empresariales, sistemas de subsistencia y limitaciones. Por tal motivo, sería apropiado formularse estrategias de desarrollo e intervenciones, que permitan a los productores ser eficientes y eco sustentables (Tamayo et al., 2014).

\section{AGRICULTURA SUSTENTABLE}

Todas las definiciones de agricultura sustentable promueven armonía ambiental, económica y social para cumplir con el significado del concepto de sustentabilidad (Zinck et al., 2005). El concepto de agricultura sustentable es una respuesta relativamente reciente a la declinación en la calidad de la base de los recursos naturales asociada con la agricultura moderna. En la actualidad, la cuestión de la producción agrícola ha evolucionado desde una forma puramente técnica hacia una más compleja, caracterizada por dimensiones sociales, culturales, políticas y económicas (Altieri, 2015).

La agricultura es sustentable cuando proporciona un rendimiento constante a largo plazo, como resultado de la optimización del sistema en su conjunto y el uso de tecnologías ecológicamente racionales (Toro et al. 2011). En los últimos años, la creciente conciencia sobre el negativo impacto ambiental, social y cultural de ciertas prácticas de la agricultura moderna ha llevado a plantear la necesidad de un cambio hacia un modelo agrícola más sustentable (Gliessman, 2001 citado por Sarandón y Flores, 2009). El enfoque de la sustentabilidad aporta una mirada ecológica sobre los procesos humanos, cuyos criterios permiten establecer que la construcción de los paisajes urbanos se relaciona con el logro de cualidades del propio espacio citadino, vinculadas al bienestar (Caquimbo y Devoto 2010).

Desde la perspectiva de las relaciones que ocurren bajo el marco de las sociedades urbanas-naturaleza, cada vez es más reconocida la importancia de la fuerte conexión entre la resiliencia y la sustentabilidad de los sistemas socioecológicos en las ciudades, muchos de ellos rurales o agroproductivos (Torres y Cedeño 2015). Echeverri (2001), indicó que las causas de la degradación del suelo están asociadas con factores de agotamiento por sobreexplotación, pero también por pérdida de los sistemas de regulación hídrica o de protección eólica.

En estos casos, la permanencia y conservación de la riqueza de los suelos no solo depende de los sistemas de productivos aplicados por los productores usuarios directos de la tierra, sino depende de factores exógenos que normalmente no son cubiertos por 
mecanismos de protección. Altieri (1999; citado por Garcés, 2011) afirmó que para la visión agroecológica la clave no está en la alta productividad, sino en la base filosófica de los principios de la agricultura sustentable, la cual incluye la concepción integral de los agro ecosistemas y el énfasis en la biodiversidad.

En este sentido, Altieri (1997; citado por Contreras, 2018) aseveró que la agricultura no solo se orienta a obtener altos rendimientos de algún producto, sino más bien a optimizar el sistema entero, puesto que las metodologías denominadas de "investigación de sistemas prediales o de finca" enfatizan la comprensión de los sistemas agrícolas tradicionales como punto de partida, evalúan los antecedentes de la zona, realizan exámenes en el terreno que incluyen entrevistas a los agricultores respecto a las características del predio y analizan acerca de por qué los agricultores emplean métodos particulares de producción. Los limitados recursos naturales (Ej: agua) no permiten una agricultura apropiada que satisfaga la seguridad alimentaria mundial. El proceso de agricultura sostenible de un país presenta alta demanda de recursos naturales lo que origina conflictos de toda índole: sociales, económicos y ecológicos, para lo cual se debe desarrollar estrategias para cubrir el crecimiento demográfico, la seguridad alimentaria, la protección a la biodiversidad y otros factores necesarios para el crecimiento del capital humano (Tamayo Manrique et al., 2014). La reducción del hambre y la pobreza se dará a través de una producción sustentable, basada en minifundio ya que será el responsable de proveer la mayor cantidad de alimentos para una población creciente (Dixon et al., 2001). El desarrollo sostenible involucra la generación de alimentos de calidad, manteniendo la fertilidad del suelo, minimizando la erosión, disminuyendo la contaminación del agua, prescindiendo del uso de agrotóxicos e incentivando los policultivos (Muñoz et al., 2016). De acuerdo a Bacon et al. (2012) los sistemas agrícolas están integrados a procesos socio ecológicos amplios que deben ser considerados en la discusión de la agricultura sustentable. El desarrollo de tecnologías agrícolas, como por ejemplo, uso de pesticidas, fertilizantes inorgánicos, maquinaria agrícola, sistemas intensivos, entre otros; se está convirtiendo en un problema ecológico en el mundo, siendo de vital importancia evaluar la eficiencia de los sistemas de producción agrícolas relacionados con su sostenibilidad (De Molina y Amate, 2010). La evaluación de la sostenibilidad de los sistemas de producción agrícolas, mediante el uso de una metodología y de indicadores, permite observar claras tendencias en el desarrollo de los sistemas productivos. La utilidad y el uso de este procedimiento metodológico se basan en que detecta puntos críticos de la sostenibilidad, establece sus causas y propone soluciones a mediano plazo. Un indicador es un signo, típicamente medible, que puede reflejar una característica cuantitativa o cualitativa, y que es importante para hacer juicios sobre condiciones del sistema actual, pasado o hacia el futuro (Bolívar, 2011).

\section{SUSTENTABILIDAD DE LOS SISTEMAS DE PRODUCCIÓN ANIMAL}

El mejoramiento genético al desarrollar poblaciones adaptadas a las condiciones de los sistemas de producción bovina del trópico (SPBT) y con la capacidad para mejorar la productividad animal al ofrecerles un mejor manejo, puede participar en la sustentabilidad de estos sistemas. Los SPBT son generalmente semi-intensivos con fuertes limitantes para este fin. Su fortaleza se basa en el uso de praderas de gramíneas, que es su principal fuente alimenticia; pero el pastoreo de estas provoca una serie de limitantes bio-ecológicas como 
el clima y la presencia de parásitos, las cuales se amplifican con los problemas socioeconómicos (Osorio y Segura, 2011). Estos últimos son más limitantes que en los sistemas de zonas templadas (Blake, 2004), que debido a las limitantes del petróleo del cual dependen presentan serios retos (Leng, 2010). Esto hace muy difícil que los SPBT alcancen la sustentabilidad (Osorio y Segura, 2011).

La definición de objetivos del mejoramiento genético, en los trópicos considerando los aspectos bioecológicos, es en sí, un problema complejo (Franklin, 1986 citado por Osorio y Segura, 2011) y al considerar a la vez los aspectos socio-económicos puede parecer una tarea imposible. Es condición básica e indispensable que la definición de objetivos del mejoramiento genético debe partir del conocimiento de los SPBT para identificar las características a mejorar. La consideración de los avances en la biología moderna y experiencias ganadas en el trópico deben apoyar los planteamientos. Así, el conocimiento de que la sobrevivencia de un animal depende de la capacidad de ajustar su metabolismo a los cambios del ambiente y de presentar reacciones inmunes en contra de organismos patógenos (Burton, 2007 citado por Osorio y Segura, 2011). La producción de los animales se mejora dentro de la potencialidad que la sustentabilidad del sistema permita y deben responder productivamente al mejoramiento de los sistemas de producción bovina tropical (Osorio y Segura, 2011).

La autosuficiencia alimentaria, que se alcanza cuando se satisfacen las necesidades alimenticias mediante la producción local, generalmente suele ser un objetivo de las políticas nacionales. Favorecer un modo de vida compasivo hacia los animales y asegurar que las personas tengan los medios para alimentarse de manera satisfactoria y sustentable en el tiempo, mediante el uso racional de los recursos naturales (FAO, 2000).

Una granja integral se refiere a la combinación de sistemas agrícolas y pecuarios, logrando un eficiente uso de los recursos existentes en un determinado lugar. La producción de estas granjas está destinada principalmente para consumo humano y los excedentes son utilizados para la alimentación animal. Las granjas integrales articulan diversos subsistemas, los que trabajando en conjunto permiten la sustentabilidad de la producción agropecuaria con prácticas más amigables con el medio ambiente, resguardando la soberanía alimentaria de la población y mejorando la calidad de vida de los campesinos. Las tendencias hacia la sostenibilidad de las granjas se deben al mejor uso del suelo. Así como, al implantar sistemas de producción adaptados a cada tipo de suelo para asegurar la rentabilidad y permanencia de los mismos, consiguiendo el mayor rendimiento posible para cada sistema de producción agropecuaria. La urgencia por producir alimentos para una población creciente no debe ser el paradigma que refuerce el imperativo para obtener la máxima rentabilidad por unidad de superficie, y que genera una visión del mundo rural orientada en obtener una mayor rentabilidad a expensas de los atributos y valores de la vida rural (Muñoz et al., 2016).

Un sistema es sustentable a medida que disminuye el periodo de rotación a lo largo del tiempo. Todas las fincas tienen menos del 10\% de la superficie del suelo cubierta. Se considera que un sistema es sustentable a medida que aumenta la cobertura del suelo con algún tipo de cultivo vegetal, disminuyendo de este modo el impacto de las lluvias y el sol en la superficie y, por otro lado, aportando materia orgánica al suelo (Andrade, 2017). Una 
política del ser inspira el clamor por el reconocimiento de los derechos a calidad de vida y la diversidad cultural de los pueblos, que brinda un valor agregado a cada comunidad para forjar su propio futuro, transformando y reconstruyendo las relaciones sociales y productivas con la naturaleza bajo la perspectiva de la sustentabilidad del desarrollo. Aun en el interior de la globalización se forjan nuevas identidades donde se conjugan diferentes regímenes históricos y epistémicos para que nuevos saberes se articulen con las ciencias y las tecnologías modernas (Leff et al., 2002 citados por Olmos y González, 2013).

\section{EVALUACIÓN DE LA SUSTENTABILIDAD}

En 1983, la Comisión Mundial de Ambiente y Desarrollo (WCED, World Comisión of Environment and Development) identifica por primera vez la importancia de evaluar cualquier acción o iniciativa humana desde tres enfoques: el económico, el ambiental y el social, expresando las relaciones directas entre la economía y la ecología, con la intención de que gobiernos y sociedades puedan actuar responsablemente respecto al deterioro ambiental (Calvente, 2007). Masera et al. (1999; citados por Merma y Julca, 2012) señalaron que la evaluación de sustentabilidad se lleva a cabo en: (a) sistemas de manejo específicos en un lugar geográfico y bajo un contexto social; (b) una escala espacial (parcela, unidad de producción o cuenca) previamente determinada; y (c) una escala temporal, aseverando también que los esfuerzos por evaluar las condiciones de sustentabilidad de los sistemas de producción han permitido el desarrollo de metodologías como el Marco MESMIS o Marco para la Evaluación de Sistemas de Manejo incorporando Indicadores de Sustentabilidad; en concordancia con la propuesta metodológica de Sarandón y Flores (2009) que, entre otros aspectos establece criterios para la caracterización del sistema, la cuantificación de los indicadores y la determinación de los puntos críticos. La estimación de "indicadores de sustentabilidad" es una manera de evaluar estos efectos y puede ayudar a la formulación de políticas agropecuarias que respeten el ambiente y sean socialmente aceptables (Bell y Morse, 2008); ya anteriormente Zinck y Farshad (1995) indicaron que la sustentabilidad no puede medirse directamente, por ser un concepto, requiriéndose indicadores adecuados para determinar el nivel y la duración de la sustentabilidad.

En general, la mayoría proponen, la evaluación de las dimensiones: ecológica, económica y social o sociocultural (Blandi et al., 2015). Para evaluar la sustentabilidad de cada dimensión señalada, hay métodos de análisis multicriterio por medio de la evaluación de un conjunto de indicadores para conocer los puntos críticos de la sustentabilidad de los agroecosistemas (Meza y Julca, 2015). En América Latina existen experiencias de evaluación de la sustentabilidad de las actividades agrícolas o de determinados sistemas de gestión de recursos naturales (Vega et al., 2015). Los métodos aplicados van desde la evaluación de temas específicos, tales como la calidad de los suelos (Arzeno, 2006) o el impacto de los pesticidas, hasta sistemas más complejos constituidos por numerosos indicadores, como el MESMIS (Método de Evaluación de la Sustentabilidad Mediante Indicadores de Sustentabilidad) (Astier et al., 2008).

Si bien muchos de estos métodos se presentan como enfoques integrales para la evaluación agropecuaria, en general se puede observar un claro sesgo hacia la estimación de variables productivas o económicas. En el MESMIS; por ejemplo, alrededor del 80\% de los indicadores utilizados se relacionan con cuestiones biofísicas o económicas del 
establecimiento (Vega et al., 2015), ya Castaño (2013) había indicado que el desarrollo sostenible propone tres pilares fundamentales: Lo económico, lo social y lo ambiental; al unir los tres pilares emergen los términos de sostenibilidad, lo equitativo, lo soportable y lo viable. Debido a que las razones de adopción de tecnología están determinadas en gran parte por la conveniencia económica (definida mediante un análisis costo beneficio) que desconoce costos ambientales y sociales, los sistemas de cultivo bajo cubierta son más rentables, pero menos sustentables (ecológicamente) que los sistemas al aire libre (Blandi et al., 2015). Las condiciones evaluadas para la sustentabilidad ambiental, económica y social, refieren un nivel de sustentabilidad intermedio por la preponderancia de indicadores que se orientan a la conservación y mejor aprovechamiento de los recursos productivos con prácticas agroecológicas, la diversificación de las actividades productivas y la comunicación intergeneracional de prácticas sustentables locales al involucrar a la familia en las labores agrícolas cotidianas (Meza y Julca, 2015). La participación y el apoyo del público en el uso de indicadores es altamente deseable, como uno de los elementos o requisitos generales de la participación de la sociedad en su conjunto en la búsqueda del desarrollo sustentable (Gaviño, 2010). El Desarrollo Sustentable tiene como premisa, el conocimiento más profundo de las interacciones entre el sistema económico y los sistemas biofísicos, que fundamentan las decisiones acordes a criterios de carácter ecológico y de viabilidad a largo plazo, esto se soporta con cuadros académicos y de investigación, así como de profesionales capacitados, que logren articular el quehacer de las universidades, centros de investigación, colegios de profesionales y asociaciones empresariales con las necesidades de la reconversión de la industria, los servicios y las actividades primarias.

\section{CONCLUSIONES}

La etnicidad de los productores asociado al conocimiento y prácticas tradicionales agrícolas, se orientan a la conservación y preservación de los recursos naturales, fundado en el temor y respeto en la utilización y explotación racional de los recursos naturales. Es una urgencia en América Latina, el desarrollo de sistemas de crianza que generen animales con alta resistencia al ambiente y potencial productivo para utilizar con mayor eficiencia los recursos disponibles en los sistemas de producción animal. El uso indiscriminado de los agroquímicos y las tecnologías aplicadas en los sistemas agropecuarios están causando a pasos acelerados el deterioro de los suelos agrícolas; todo ello, trae como consecuencia la contaminación del medio ambiente, en donde la agricultura y la ganadería se están convirtiendo en un problema ecológico, por lo que se requiere evaluar la eficiencia de los sistemas de producción agrícolas en relación con su sustentabilidad. El sistema tradicional campesino es aparentemente insostenible, mientras que las granjas de producción con enfoque integral tienen mejores oportunidades de desarrollo en el tiempo, ya que estas granjas integrales incorporan alternativas productivas, articulan diversos subsistemas, los que trabajando en conjunto permiten una mayor sustentabilidad de la producción agropecuaria y una mejora de la calidad de vida de los campesinos. La intensificación de los sistemas agropecuarios puede ser positiva siempre en cuando se desarrolle de manera sustentable; es decir, atendiendo en el presente las necesidades humanas, pero cuidando los recursos para las generaciones futuras, con rentabilidad económica y respetando el medio ambiente. De lo contrario se excedería los límites de tolerancia, tanto ambiental 
como social, ello debido a los cambios ecológicos, demográficos, culturales y económicos que una intensificación no planificada puede causar.

\section{REFERENCIAS BIBLIOGRÁFICAS}

Albicetti M., Brasesco R. y Chiappe M. (2009). Propuesta de indicadores para evaluar la sustentabilidad predial en agroecosistemas agrícola-ganaderos del litoral del Uruguay. Agrociencia (2009) Vol XIII Nº 1 pág. 48 - 68

Altieri M. A. (2015). Agroecología: principios y estrategias para diseñar sistemas agrarios sustentables. https://www.researchgate.net/publication/255668549

Altieri M.A., Funes M.F., Petersen P., Tomic T. y Medina Ch. (2011). Sistemas agrícolas ecológicamente eficientes para los pequeños agricultores. Foro Europeo de Desarrollo Rural 2011. Palencia, España. 27 p.

Andrade C.K. (2017). Análisis Sustentable de las Fincas de Brócoli (Brassica oleracea L. var. italica) en Santa Rosa de Quives, Lima, Perú. Ecología Aplicada, 16(2), 2017.

Arzeno J.L. (2006). Empleo de indicadores de sostenibilidad en sistemas extensivos agrícolas del NOA. En: Actas de las Primeras Jornadas Interdisciplinarias de estudios agrarios y agroindustriales del NOA. Instituto Nacional de Tecnología Agropecuaria (INTA), Salta. http://appweb.inta.gov.ar/w3/prorenoa/zonadescarga/Sist_prod_sost/ 54_Indicadores $\% 20$ sostenibilidad $\% 20$ en $\% 20$ Sist $\% 20$ Ext.pdf

Astier M., Masera O.M., Galván Y. (2008). Evaluación de la sustentabilidad. Un enfoque dinámico y multidimensional. Mundi-Prensa, México D.F.

Bacon C., Getz C., Kraus S., Montenegro M. and Holland K. (2012). The social dimensions of sustainability and change in diversified farming systems. Ecology and Society. 17(4):1-41.

Bautista J.A. y Smit M.A. (2012). Sustainability and agriculture in the "mezcal region" of Oaxaca. Revista Mexicana de Ciencias Agrícolas Vol.3 Núm.1. $1^{\circ}$ de enero 29 de febrero, 2012 p. 5-20.

Bell B. y Morse S. (2008). Sustainability indicators: measuring the immeasurable? 2 ed. Earthscan Publications Ltd., Londres.

Blake R. (2004). Dairy cattle response in difficult environments. ProceedingsXL Reunión de Investigación Pecuaria. 2nd Simposium Internacional sobre transferencia de tecnología agropecuaria. Mérida, Yucatán, México. 119-127.

Blandi M.L., Sarandón S.J., Flores C.C., Caquimbo I.V., Devoto C. (2015). Evaluación de la sustentabilidad de la incorporación del cultivo bajo cubierta en la horticultura platense. Rev. Fac. Agron. Vol 114 (2): 251-264.

Bocero S. (2002). Cultivos protegidos y problemas ambientales: un estudio de la horticultura Marplatense en la década del Noventa. [Tesis]. FLACSO. Universidad Nacional de Mar de Plata. Argentina. 
Bolívar H. (2011). Metodologías e indicadores de evaluación de sistemas agrícolas hacia el desarrollo sostenible. Red de Revistas Científicas de América Latina y el Caribe, Redalyc 8(1), 1-18.

Contreras S.E. (2018). Sustentabilidad de la producción de papa en la región Lima. [Tesis] UNALM. Lima, Perú.

De Molina M.L.G. y Amate J.I. (2010). Agroecología y decrecimiento. Una alternativa sostenible a la configuración del actual sistema agroalimentario español. Revista de Economía Crítica, 10: 113-137.

Dixon J., Gulliver A., Gibbon D. (2001). Sistemas de producción agropecuaria y pobreza: cómo mejorar los medios de subsistencia de los pequeños agricultores en un mundo cambiante. Malcolm Hall. FAO.

Echeverri R. (2001). La nueva ruralidad en América Latina y el Caribe. Instituto interamericano de cooperación para la agricultura (IICA). Centro internacional de desarrollo rural (CIDER). Mimeo.

FAO (2014). Metas e indicadores para la agenda de desarrollo post-2015 y los objetivos de desarrollo sostenible. FAO, Roma.

FAO (2000). Mejorando la nutrición a través de huertos y granjas familiares. Manual de capacitación para trabajadores de campo en América Latina. FAO Roma, Italia.

Garcés S. (2011). Bienestar y sustentabilidad en el medio rural. Edc. Abya-Yala. Quito, Ecuador. P.21.

Gaviño M. (2010). Indicadores Ambientales y su aplicación. UNESCO. Santiago, Chile. 44p.

Leng R. A. (2010). The impact of resource depletion is being overshadowed by the threat of global warming. Livestock Research for Rural Development. Retrieve June 15, 2010 from http://www.lrrd.org/lrrd22/2/leng.htm

Martínez R. (2009). Sistemas de producción agrícola sostenible. Rev. Tecnología en Marcha, 22 (2): 23-29.

Merma I. y Julca A. (2012). Caracterización y evaluación de la sustentabilidad de fincas en alto Urubamba, Cusco, Perú. Ecología Aplicada, 11(1), 2012.

Meza Y. y Julca A. (2015). Sustentabilidad de los sistemas de cultivo con yuca (Manihot esculenta Crantz) en la subcuenca de Santa Teresa, Cusco. Ecología Aplicada, 14(1), 2015.

Muñoz M., Artieda J., Espinoza S., Curay S., Pérez M., Núñez O., Mera R., Zurita H., Velástegui G., Pomboza P., Carrasco A., Barros M. (2016). Granjas Sostenibles: integración de sistemas agropecuarios. Tropical and Subtropical Agroecosystems, vol. 19, núm. 2, mayo-agosto, 2016, pp. 93-99 Universidad Autónoma de Yucatán Mérida, Yucatán, México.

Olmos M.A. y González S.W. (2013). El valor de la sustentabilidad. Ciencia y Agricultura, vol. 10, núm. 1, enero-junio, 2013, pp. 91-100. 
Osorio M.M. y Segura J.C. (2011). Sustentabilidad de los sistemas de producción bovina en el trópico: mejoramiento genético. Livestock Research for Rural Development 23 (8) 2011.

Sarandón S.J. y Flores C.C. (2009). Evaluación de la sustentabilidad en agroecosistemas: una propuesta metodológica. Agroecología 4:19-28.

Tamayo J., Martínez E., Monforte G., Munguía A., Ruiz A. (2014). La agroecología como propuesta de modelo de producción aplicado al cultivo de chile habanero en peto, Yucatán. Revista Mexicana de Agronegocios, 18(35): 969-978.

Toro P., García A., Gómez A.G., Acero R., Perea J. y Rodríguez V. (2011). Sustentabilidad de Agroecosistemas. Archivos de Zootecnia 60: 15-39.

Torres P. y Cruz J. (2019). Procesos urbanos y sistemas socio ecológicos. Trayectorias sustentables de la agricultura de chinampa en Ciudad de México. Letras Verdes. Revista Latinoamericana de Estudios Socio ambientales N. ${ }^{\circ}$ 25, marzo de 2019, pp. 168-189.

Torres P. y Coords A.C. (2015). Ecourbanismo y habitabilidad regional. Contribuciones de América Latina. México: UAM-X.

Vega M.L., Iribarnegaray M.A., Hernández M.E., Arzeno J.L., Osinaga R., Zelaayán A.L., Fernández D.R., Serrano F.H., Volante J.N. y Seghezzo L. (2015). Un nuevo método para la evaluación de la sustentabilidad agropecuaria en la provincia de Salta, Argentina. RIA / Vol. 41 / N² 2.

Zinck J. A., Berroterán J. L, Farshad A., Moameni A., Wokabi S., Van Ranst E. (2005). La sustentabilidad agrícola: un análisis jerárquico. Gaceta Ecológica, núm. 76, julio-septiembre, 2005, pp. 53-72. sistemas de producción agropecuaria. Puriq, 1(02), 198-207. https://doi.org/10.37073/puriq.1.02.31 